\title{
Insulin Resistance and Cardiovascular Risks in Different Groups of Hemodialysis Patients: A Multicenter Study
}

\author{
Tuyen Van Duong ${ }^{D},{ }^{1}$ Chun-Kuang Shih, ${ }^{1}$ Te-Chih Wong, ${ }^{2}$ Hsi-Hsien Chen $\left(\mathbb{D},{ }^{3,4}\right.$ \\ Tso-Hsiao Chen, ${ }^{4,5}$ Yung-Ho Hsu, ${ }^{4,6}$ Sheng-Jeng Peng, ${ }^{7}$ Ko-Lin Kuo, ${ }^{8}$ Hsiang-Chung Liu, \\ En-Tzu Lin, ${ }^{10}$ Chien-Tien Su $\mathbb{D},{ }^{11,12}$ and Shwu-Huey Yang $\mathbb{D}^{1,13,14}$ \\ ${ }^{1}$ School of Nutrition and Health Sciences, Taipei Medical University, Taipei, Taiwan \\ ${ }^{2}$ Department of Nutrition and Health Sciences, Chinese Culture University, Taipei, Taiwan \\ ${ }^{3}$ Department of Nephrology, Taipei Medical University Hospital, Taipei, Taiwan \\ ${ }^{4}$ School of Medicine, Taipei Medical University, Taipei, Taiwan \\ ${ }^{5}$ Department of Nephrology, Taipei Medical University- Wan Fang Hospital, Taipei, Taiwan \\ ${ }^{6}$ Division of Nephrology, Department of Internal Medicine, Taipei Medical University-Shuang Ho Hospital, Taipei, Taiwan \\ ${ }^{7}$ Division of Nephrology, Cathay General Hospital, Taipei, Taiwan \\ ${ }^{8}$ Division of Nephrology, Taipei Tzu-Chi Hospital, Taipei, Taiwan \\ ${ }^{9}$ Department of Nephrology, Wei Gong Memorial Hospital, Miaoli, Taiwan \\ ${ }^{10}$ Department of Nephrology, Lotung Poh-Ai Hospital, Yilan, Taiwan \\ ${ }^{11}$ School of Public Health, Taipei Medical University, Taipei, Taiwan \\ ${ }^{12}$ Department of Family Medicine, Taipei Medical University Hospital, Taipei, Taiwan \\ ${ }^{13}$ Research Center of Geriatric Nutrition, Taipei Medical University, Taipei, Taiwan \\ ${ }^{14}$ Nutrition Research Center, Taipei Medical University Hospital, Taipei, Taiwan
}

Correspondence should be addressed to Chien-Tien Su; ctsu@tmu.edu.tw and Shwu-Huey Yang; sherry@tmu.edu.tw

Received 27 December 2018; Revised 20 February 2019; Accepted 16 May 2019; Published 11 June 2019

Academic Editor: Koichiro Wada

Copyright (C) 2019 Tuyen Van Duong et al. This is an open access article distributed under the Creative Commons Attribution License, which permits unrestricted use, distribution, and reproduction in any medium, provided the original work is properly cited.

Background. To investigate the association between insulin resistance (IR) and cardiovascular disease (CVD) risks among hemodialysis patients. Methods. We conducted a cross-sectional study between 2013 and 2017, on 384 hemodialysis patients from seven hospital-based-dialysis centers. HOMA-IR is classified according to median value. The CVD risks were defined by the K/DOQI Guidelines. Logistic regression analysis was used. Results. Patients' age was $60.9 \pm 11.8,58.1 \%$ men, and $40.3 \%$ overweight/obese. The median of HOMA-IR was 5.4, 82.8\% high systolic blood pressure, and $85.7 \%$ hyperhomocysteinemia. In multivariate analysis, IR was significantly associated with higher odds of low high-density lipoprotein cholesterol, high triglyceride, and impaired fasting glucose in groups of normal weight, overweight/obese, nondiabetes, diabetes, and overall sample. IR linked with elevated high-sensitive $\mathrm{C}$-reactive protein in normal weight patients (odd ratio, $\mathrm{OR}=2.21,95 \%$ confidence interval, $1.16-4.22, \mathrm{p}$ $<.05)$, with hypoalbuminemia in normal weight patients ( $\mathrm{OR}=8.31,95 \% \mathrm{CI}, 2.35-29.37, \mathrm{p}<.01)$, in nondiabetes patients $(\mathrm{OR}=6.59$, $95 \%$ CI, 1.81-23.95, $\mathrm{p}<.01)$, and overall sample $(\mathrm{OR}=3.07,1.51-6.23, \mathrm{p}<.01)$. Conclusions. The level of IR and prevalence of CVD risks were high in hemodialysis patients. IR was independently associated with CVD risks.

\section{Introduction}

The incidence and prevalence of treated end-stage renal disease (ESRD) have been steadily increased over the past decades across countries. In 2014, Taiwan has reported the highest number of treated ESRD with 455 new cases and the prevalence of 3219 patients per million general population (PMP). Taiwan has also experienced the highest number of maintenance hemodialysis in the world with 3093 patients PMP, 90\% of them receiving in-center hemodialysis [1]. 
Cardiovascular disease (CVD) has been reported as the leading cause of death and disability all over the world. In 2013, CVD accounted for about 17 million deaths and 329 million disability adjusted life-years lost [2]. In ESRD patients, the cardiovascular cause of death is 10-20 times higher in the healthy population and accounted for more than half of all death [3].

Insulin resistance (IR) is with high prevalence in the ESRD patients [4]. IR its self is a risk for CVD and strongly associates with other CVD risks (dyslipidemia, hypertension, and inflammation) through several pathophysiologic mechanisms, which is well documented [5]. In ESRD patients undergoing hemodialysis, the cardiovascular risks worsen the arterial stiffness which contributes to the development of cardiovascular events and diseases [6]. IR is the anterior consequence of obesity [7]. In ESRD patients, IR then links to dyslipidemia, impaired fasting glucose, and cardiovascular risks and events [5, 8, 9]. In empirical researches, IR is closely associated with cardiovascular risks such as obesity, hypertension, and dyslipidemia [10], anemia [4], inflammation [11, 12], and echocardiography parameters [12]. In turn, IR significantly predicts cardiovascular diseases and mortality in ESRD patients [13-16]. Therefore, assessment of IR is critically important work for nephrologist and nurses to follow up patients and have appropriate interventions.

The ESRD has created a heavy burden for the healthcare system all around the world over the past decade [1]. However, the number of clinicians has not adequately increased to meet the greater demand for renal treatment [17]. The early detection of the IR and its associated factors might contribute to prevent CVD risks and reduce the burden. On the other hand, improving IR might be an important therapeutic target and contribute to better health outcomes in hemodialysis patients $[18,19]$. This study was to assess the prevalence and explore the association between IR and CVD risk factors among ESRD patients undergoing hemodialysis.

\section{Materials and Methods}

2.1. Study Design. A clinical cross-sectional study was conducted between September 2013 and April 2017 in seven dialysis centers in Taiwan. A total of 384 hemodialysis patients were recruited from Taipei Medical University Hospital (55 patients collected from September to December 2013; 42 patients collected from November 2016 to January 2017); Taipei Medical University, Wan Fang Hospital (51 patients collected from April to May 2014); Taipei Medical University, Shuang Ho Hospital (39 patients collected in December 2014); Cathay General Hospital (41 patients collected in March 2016); Taipei Tzu-Chi Hospital (57 patients collected in November 2016); Wei-Gong Memorial Hospital (59 patients collected from February to March 2017); and Lotung Poh-Ai Hospital (50 patients collected in April 2017).

2.2. Hemodialysis Patients and Data Sources. We included patients aged above 20 years, receiving thrice-weekly hemodialysis treatment for at least 3 months and adequate dialysis quality (equilibrated $\mathrm{Kt} / \mathrm{V} \geq 1.2 \mathrm{~g} / \mathrm{kg} /$ day). The exclusion criteria were patients who were diagnosed with pregnancy, amputation, hyperthyroidism, hypothyroidism, and malignancy, received tube feeding, exhibited hepatic failure or cancer, were hospitalized within one month prior to the recruitment, or were scheduled for surgery. Volume overload or edema closely linked with other clinical instability [20]. Therefore, patients with evidence of edema were excluded in the current study and in previous studies [21-23].

The eligible patients in selected hospitals signed the informed consents before conducting chart reviews and laboratory evaluations. The patients' medical records were reviewed. The blood samples were collected by licensed nurses, at the start of the first dialysis session of the week, and then analyzed in the hospital laboratory by using commercially available test kits, which was described carefully in previous studies $[24,25]$.

2.3. Insulin Resistance Index. The blood samples collected by the registered nurse were centrifuged in each hospital laboratory. The serum was separated and kept in the icepack, then sent to the laboratory in Taipei Medical University Hospital for serum insulin analysis. Therefore, all samples were analyzed with the same commercial kit. The homeostatic model assessment of insulin resistance index (HOMA-IR) is used to assess IR. The index is calculated using the formula developed by Matthews et al. [26]:

$$
\begin{aligned}
\text { HOMA-IR }= & \text { fasting plasma insulin }(\mu \mathrm{U} / \mathrm{mL}) \\
& \times \frac{\text { fasting plasma glucose }(\mathrm{mg} / \mathrm{dL})}{405} .
\end{aligned}
$$

Patients were separated into two groups based on the median value as the nonnormal distribution of HOMA-IR; this method was applied in previous studies [14, 27].

2.4. Cardiovascular Risks. The traditional and nontraditional CVD risks were described in the previous study [22] and the current study with the details below. In the present study, more factors were assessed and reported, such as physical activity, medical history (diabetes, hypertension, and cardiovascular disease), and other biochemical parameters (blood urea nitrogen, uric acid, creatinine, and fasting plasma insulin).

2.4.1. Traditional CVD Risk Factors. The traditional risks of cardiovascular diseases include factors which were mentioned in previous studies $[28,29]$. (1) Hypertension: systolic blood pressure $(\mathrm{SBP}) \geq 130 \mathrm{mmHg}$ and diastolic blood pressure (DBP) $\geq 85 \mathrm{mmHg}$ [30]; (2) impaired fasting glucose (IFG): patients diagnosed with type 2 diabetes mellitus or fasting plasma glucose (FPG) $\geq 100 \mathrm{mg} / \mathrm{dL}$ [30]; (3) dyslipidemia including high serum triglyceride (TG) level at $\mathrm{TG} \geq 150 \mathrm{mg} / \mathrm{dL}$, low level of serum high-density lipoprotein cholesterol (HDL-C) at $<40 \mathrm{mg} / \mathrm{dL}$ in men and $<50$ $\mathrm{mg} / \mathrm{dL}$ in women, high level of serum low-density lipoprotein cholesterol (LDL-C) at $\geq 100 \mathrm{mg} / \mathrm{dL}$, and high serum total cholesterol at $\mathrm{TC} \geq 200 \mathrm{mg} / \mathrm{dL}$ [31].

2.4.2. Nontraditional/Novel CVD Risk Factors. Anemia: the targeted hemoglobin $(\mathrm{Hb})$ level should be $11 \mathrm{~g} / \mathrm{dL}$ or greater, 
as moderately strong recommended by The National Kidney Foundation Kidney Disease Outcomes Quality Initiative (K/DOQI) Work Group [32]. Anemia is classified as $\mathrm{Hb}<11$ $\mathrm{g} / \mathrm{dL}$. Mineral metabolism abnormalities: albumin-corrected calcium $=$ total calcium $(\mathrm{mg} / \mathrm{dL})+0.8 \times(4.0-$ serum albumin in $\mathrm{g} / \mathrm{dL}$ ) [33]. Corrected calcium and phosphate levels at each time were used to calculate calcium-phosphate product ( $\mathrm{Cax}$ $\left.\mathrm{PO}_{4}\right)$. The serum calcium $(\mathrm{Ca})$ is classified into low level $(\mathrm{Ca}<$ $8.4 \mathrm{mg} / \mathrm{dL}$ ), normal level (Ca 8.4- $9.5 \mathrm{mg} / \mathrm{dL}$ ), and high (Ca > $9.5 \mathrm{mg} / \mathrm{dL})$. The serum phosphate $\left(\mathrm{PO}_{4}\right)$ is also classified into low level (PO4<3.5 mg/dL), normal $\left(\mathrm{PO}_{4} 3.5-5.5 \mathrm{mg} / \mathrm{dL}\right)$, and high (PO4 $>5.5 \mathrm{mg} / \mathrm{dL})$. Calcium-phosphate product is classified into normal $\left(\mathrm{Cax} \mathrm{PO}_{4}<55 \mathrm{mg}^{2} / \mathrm{dL}^{2}\right)$ and high $(\mathrm{Ca}$ $\mathrm{x} \mathrm{PO}_{4} \geq 55 \mathrm{mg}^{2} / \mathrm{dL}^{2}$ ). In addition, intact parathyroid hormone (iPTH) is classified as normal (iPTH $150-300 \mathrm{pg} / \mathrm{mL}$ ), and high (iPTH $\geq 300 \mathrm{pg} / \mathrm{mL}$ ) [34]. Hyperhomocysteinemia is defined as total plasma homocysteine (Hcy) $>14 \mu \mathrm{mol} / \mathrm{L}$ [29]. Inflammation is defined as high sensitive C-reactive protein (hs-CRP) $>0.3 \mathrm{mg} / \mathrm{dL}$ as the risk factor for CVD [35]. The poor nutritional status is defined as serum albumin $\leq 3.5 \mathrm{mg} / \mathrm{dL}$ as applied in hemodialysis patients from 11 countries in the Dialysis Outcomes and Practice Patterns Study (DOPPS) [36]. Hyperkalemia is identified as serum potassium $\geq 5.0 \mathrm{mEq} / \mathrm{L}$ as the risk of cardiovascular mortality in hemodialysis patients [37].

2.5. Statistical Analysis. The descriptive analyses describe the patients' characteristics, insulin resistance (IR), cardiovascular disease risk factors via the mean, standard deviation, or median, interquartile range, frequency, and percentage. The independent-samples t-test, Chi-square test, and MannWhitney $U$ test were used appropriately to test the distribution of patients' characteristics, CVD risks, and HOMA-IR in different groups of body mass index (BMI) and DM. In order to carefully examine the association between IR and traditional and nontraditional risk factors, the multivariate logistic regressions are used to estimate the odd ratios. Since obesity is the most common cause of IR [7], we analyzed the association in different groups of BMI (normal weight versus overweight/obese). The associations were also analyzed in a group of patients with diabetes and nondiabetes. The analyses were adjusted for age and gender, hemodialysis vintage, Charlson comorbidity index, and body mass index (for overall sample). These adjusted factors might be the confounders as they showed the relationship with IR [3842]. All statistical analyses are performed by the SPSS for Windows v20.0 (IBM Corp., New York, USA). The significant level is set at $\mathrm{p}$ value $<.05$.

2.6. Ethical Approval. The study is approved by the Joint Institutional Review Board of Taipei Medical University (TMU-JIRB No. 201302024), which was for conducting the study in three hospitals of Taipei Medical University (Taipei Medical University Hospital, Wan-Fang Hospital, Shuang Ho Hospital), Wei-Gong Memorial Hospital, and Lotung Poh-Ai Hospital; the ethical committee of Cathay General Hospital (CGH-OP104001); and Taipei Tzu-Chi Hospital (04M11-090). All patients involved in the study have signed the informed consent statement.

\section{Results}

Of the total sample, the average age of patients was 60.9 $\pm 11.8,58.1 \%$ men, and $40.3 \%$ overweight or obese. The traditional CVD risk factors included high SBP $(82.8 \%)$, high DBP (25.5\%), high TC (16.7\%), high LDL-C (48.4\%), low HDL-C (65.9\%), high TG (40.6\%), and impaired fasting glucose $(69.5 \%)$. The nontraditional CVD risks included anemia (58.3\%), low calcium (8.3\%), high calcium $(35.2 \%)$, low phosphate $(7.0 \%)$, high phosphate $(35.4 \%)$, high calciumphosphate product (25.5\%), high intact parathyroid hormone (42.7\%), hyperhomocysteinemia (85.7\%), elevated hs-CRP (45.6\%), and low serum albumin (12.0\%; Table 1$)$.

In comparison with normal weight patients, those with overweight/obese had a higher prevalence of low HDL-C, high TG, IFG, low serum Ca, elevated $\mathrm{CaxPO}_{4}$, elevated hsCRP, and elevated HOMA-IR ( $\mathrm{p}<.05)$. On the other hand, patients with DM had a higher proportion of high SBP, low HDL-C, high TG, IFG, elevated iPTH, low serum albumin, and elevated HOMA-IR, as compared with non-DM patients $(\mathrm{p}<.05$; Table 1$)$

The results of multivariate regression analyses are shown in Tables 2 and 3. After control for age, gender, hemodialysis vintage, Charlson Comorbidity Index, and physical activity, in normal weight patients, IR was significantly associated with higher odds of low HDL-C (OR, 1.75, 95\%CI, 1.01-3.06, $\mathrm{p}<.05)$, high TG (OR, 3.41, 95\%CI, 1.78-6.53, p < .001), IFG (OR, 8.15, 95\%CI, 4.14-16.02, p < .001), elevated hsCRP (OR, 2.21, 95\%CI, 1.16-4.22, p < .05), and lower odd of hypoalbuminemia (OR, 8.31, 95\%CI, 2.35-29.37, p < .01), but with lower odds of high SBP (OR, 0.36, 95\%CI, 0.17-0.76, $\mathrm{p}<.01$ ), high PO4 (OR, 0.52, 95\%CI, 0.28-0.96, p < .05), and hyperkalemia (OR, 0.39, 95\%CI, 0.22-0.70, p <.01). In overweight/obese patients, IR was significantly linked with higher odds of low HDL-C (OR, 4.15, 95\%CI, 1.71-10.06, p < $.01)$, high TG (OR, 3.06, 95\%CI, 1.53-6.13, $\mathrm{p}<.01)$, and IFG (OR, 10.76, 95\%CI, 3.36-34.5, $\mathrm{p}<.001$ ).

After being adjusted for age, gender, hemodialysis vintage, Charlson Comorbidity Index, and physical activity, and body mass index, in non-DM patients, IR was significantly linked with higher odds of low HDL-C (OR, 2.14, 95\%CI, 1.23-3.75, $\mathrm{p}<.01$ ), high TG (OR, 3.22, 95\%CI, 1.69-6.12, $\mathrm{p}$ $<.001$ ), IFG (OR, 12.54, 95\%CI, 6.39-24.63, p <.001), and hypoalbuminemia (OR, 6.59, 95\%CI, 1.81-23.95, p <.01), but with lower odd of hyperkalemia (OR, 0.31, 95\%CI, 0.17$0.57, \mathrm{p}<.001)$. In DM patients, IR was significantly linked with higher odds of low HDL-C (OR, 3.07, 95\%CI, 1.287.33, $\mathrm{p}<.05)$ and high TG (OR, 4.29, 95\%CI, 2.05-8.98, p < .001). In overall sample, the elevated level of HOMA-IR was significantly associated with higher odds of low HDL-C (OR, 2.53, 95\%CI, 1.59-4.01, p <.001), high TG (OR, 3.58, 95\%CI, 2.25-5.69, $\mathrm{p}<.001$ ), IFG (OR, 7.99, 95\%CI, 4.50-14.18, p < .001 ), and hypoalbuminemia (OR, 3.07, 95\%CI, 1.51-6.23, p $<.01$ ), but with lower odd of hyperkalemia (OR, 0.56, 95\%CI, 0.36-0.88, $\mathrm{p}<.05$; Tables 2 and 3 ).

\section{Discussion}

The level of IR is higher in overweight/obese and DM patients than in normal weight and non-DM patients in the current 


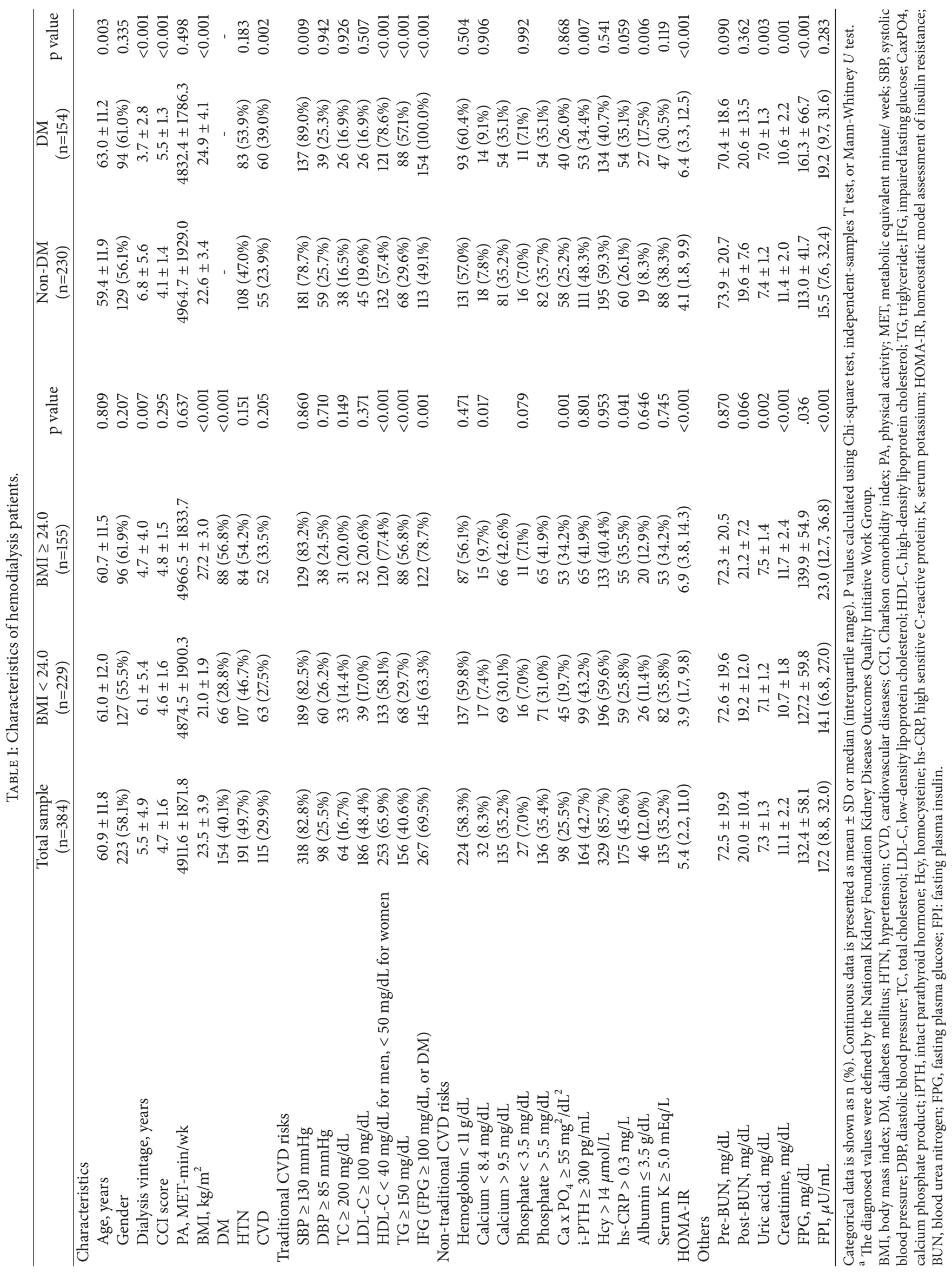




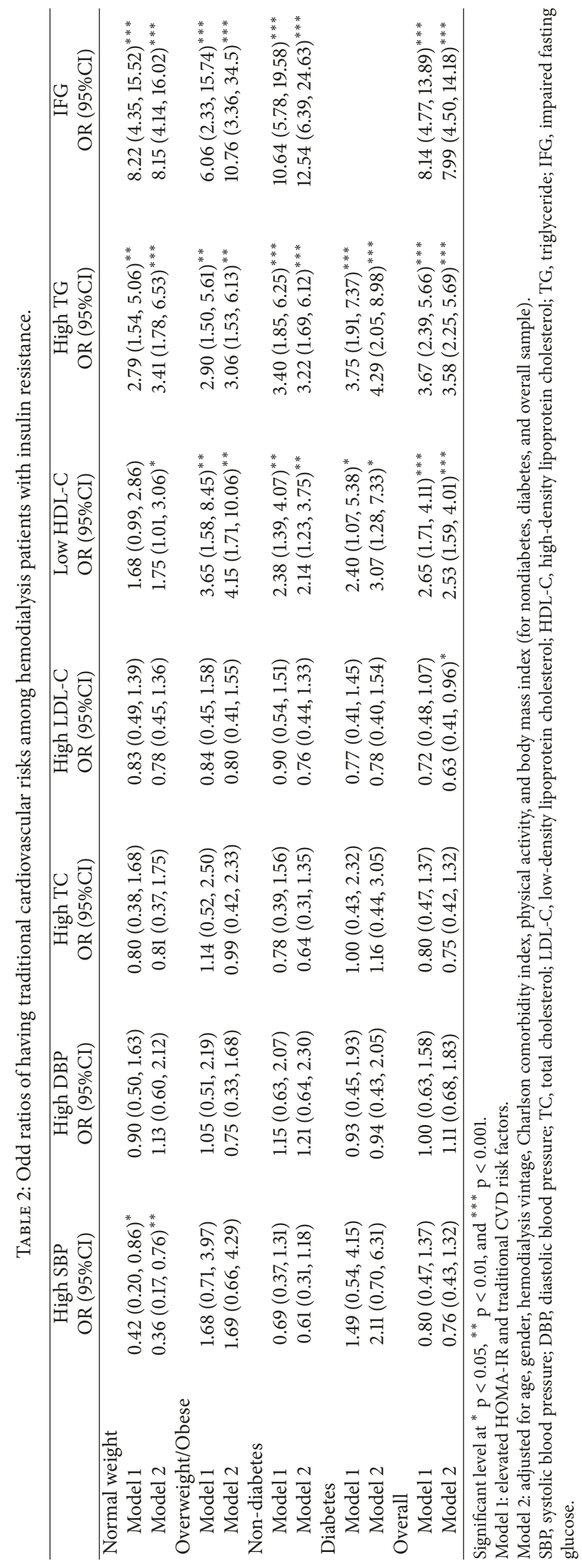




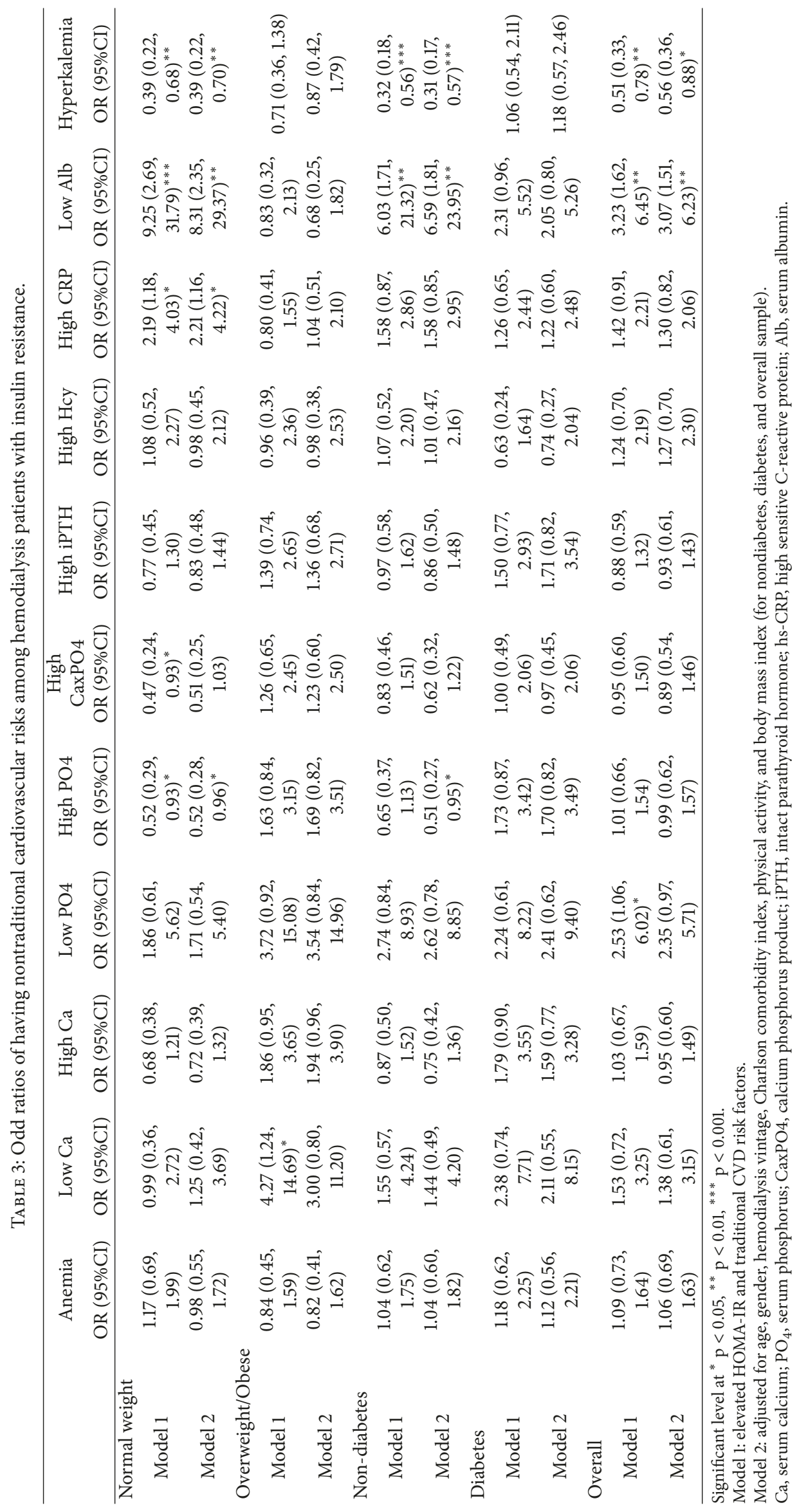


study. Obesity is also reported as the most common cause of IR previously [7].

In regard to traditional CVD risks, IR is significantly associated with a higher prevalence of dyslipidemia such as low HDL-C, high TG in the current study. The finding was also found in both the general population [43] and hemodialysis patients [9]. In the previous study, IR was found to reduce HDL-C in hemodialysis patients [9]. On the other hand, IR was also associated with higher likelihood of having impaired fasting glucose in all groups of patients, independent of age, gender, hemodialysis vintage, Charlson comorbidity index, Physical activity, and body mass index. The IR was welldocumented as the immediate factor between obesity and impaired fasting glucose and cardiovascular diseases in the literature $[5,8]$. Therefore, early interventions at stages of obesity or IR are extremely important to prevent and mitigate the adverse consequences of CVD risks, such as dietary intake, physical activities, and medication [5].

Regarding the nontraditional risks, the prevalence of elevated hs-CRP was higher in overweight/obese patients than those with normal weight. A previous study has also reported that patients with central obesity had higher hs-CRP level than those without [44]. The level of hs-CRP was not significantly differed among HD patients with and without DM. This was also found in HD patients in Japan that hsCRP was similar between HD patients with DM, HD patients with metabolic syndrome (MS), and those with neither DM nor MS [9]. In addition, the prevalence of malnutrition in the current study was with $12.0 \%$ hypoalbumin; it is much lower than in the previous study conducted in Turkey with $44.1 \%$ hypoalbumin [45].

In the current study, there is also no significant association between IR and hs-CRP in patients with DM or nonDM patients or overweight/obese, but it existed in normal weight patients. The association between IR and hs-CRP was found in the overall sample in Turkey $[11,12]$ and in 598 overweight/obese patients in Spain [44]. On the other hand, the association between IR and hypoalbumin was found in hemodialysis patients with normal weight, and non-DM, and overall sample. This was also shown in a previous study [12]. It is important to take into account the evaluation of IR level, hsCRP, and serum albumin, in order to prevent and intervene against CVD risks and diseases.

There were some contradictory findings between the current study and the previous one. Firstly, the IR associated with a lower likelihood of having high SBP in the current study which was in contrast with the previous finding [10]. Next, IR was related to a lower likelihood of hyperkalemia in normal weight, non-DM, and overall sample. This happened as the result of kalemia lowering treatment among hemodialysis patients. Finally, in normal weight, and non-DM sample, IR was associated with a lower likelihood of hyperphosphatemia in the current study. Hyperphosphatemia and high level of $\mathrm{CaxPO}_{4}$ combination were found to link with higher allcause mortality in hemodialysis patients [46].

The study was of a cross-sectional nature; the causal relationship, therefore, cannot be generated. The interpretation of results should be cautious. The data related to supplement intake and medication was not explored. Therefore, the association between IR and some CVD risks was not well explained. Smoking is known as a major traditional cardiovascular risk factor. It is reported that $85.1 \%$ of patients were nonsmokers [47]. In the United State, based on data of USRDS, $6.2 \%$ of dialysis patients were smokers [48]. Therefore, we did not collect the data on smoking status in the current study. Future longitudinal, case-control, or intervention studies are encouraged to carefully examine the association.

\section{Conclusions}

The insulin resistance (IR) and CVD risks were common in hemodialysis patients. IR was associated with a higher prevalence of dyslipidemia (low HDL-C, high TG), impaired fasting glucose, elevated hs-CRP, and hypoalbuminemia. Addressing the assessment and treatment of IR and CVD risks in clinical practice could help with improving the hemodialysis outcomes.

\section{Data Availability}

Since the dataset contains sensitive and identifying information, any modification or deidentification on the dataset is restricted. The authors confirm that the data is available upon request. Requests may be sent to the corresponding author, Shwu-Huey Yang (sherry@tmu.edu.tw).

\section{Disclosure}

The funder had no role in the decision to collect data, data analysis, or reporting of the results. The abstract was presented at the Annual Dialysis Conference 2018, Mar 3-6, 2018, Orlando, Florida.

\section{Conflicts of Interest}

The authors had no conflicts of interest relevant to this article to be disclosed.

\section{Authors' Contributions}

Tuyen Van Duong consulted a statistician, analyzed and interpreted the data, and drafted the manuscript. Chun-Kuang Shih, Te-Chih Wong, Hsi-Hsien Chen, Tso-Hsiao Chen, Yung-Ho Hsu, Sheng-Jeng Peng, Ko-Lin Kuo, Hsiang-Chung Liu, and En-Tzu Lin contributed to conception and design, acquisition of data, and discussion. Chien-Tien Su and ShwuHuey Yang contributed to the overall conception and design and critically reviewed the manuscript. All authors read and approved the final version of the manuscript.

\section{Acknowledgments}

The authors thank medical staff and patients from Taipei Medical University Hospital, Wan-Fang Hospital, Shuang Ho Hospital, Cathay General Hospital, and Taipei Tzu-Chi 
Hospital, Wei-Gong Memorial Hospital, and Lutong PohAi Hospital. The authors also thank Chi-Sin Huang, I-Hsin Tseng, Yi-Wei Feng, and Tai-Yue Chang for helping with data collection. The research was funded by the Ministry of Science and Technology in Taiwan (NSC-102-2320-B-038026; MOST 105-2320-B-038-033-MY3).

\section{References}

[1] United States Renal Data System, "International comparisons," The 2016 Annual Data Report: Epidemiology of kidney disease in the United States: Volume 2 - End-stage Renal Disease (ESRD) in the United States, USRDS Coordinating Center: National Institutes of Health, National Institute of Diabetes and Digestive and Kidney Diseases, 2016.

[2] GBD 2013 Mortality and Causes of Death Collaborators, "Global, regional, and national age-sex specific all-cause and cause-specific mortality for 240 causes of death, 1990-2013: a systematic analysis for the Global Burden of Disease Study 2013," The Lancet, vol. 385, no. 9963, pp. 117-171, 2015.

[3] United States Renal Data System, 2016 USRDS Annual Data Report: Epidemiology of Kidney Disease in the United States, USRDS Coordinating Center: National Institutes of Health, National Institute of Diabetes and Digestive and Kidney Diseases, Bethesda, Md, USA, 2016.

[4] D. Sit, A. K. Kadiroglu, M. E. Yilmaz, I. H. Kara, and B. Isikoglu, "The prevalence of insulin resistance and its relationship between anemia, secondary hyperparathyroidism, inflammation, and cardiac parameters in chronic hemodialysis patients," Renal Failure, vol. 27, no. 4, pp. 403-407, 2005.

[5] D. J. Rader, "Effect of insulin resistance, dyslipidemia, and intraabdominal adiposity on the development of cardiovascular disease and diabetes mellitus," American Journal of Medicine, vol. 120, supplement 1, no. 3, pp. S12-S18, 2007.

[6] B. El Ghoul, Y. Daaboul, S. Korjian et al., "Etiology of endstage renal disease and arterial stiffness among hemodialysis patients," BioMed Research International, vol. 2017, Article ID 2543262, 6 pages, 2017.

[7] A. M. F. Johnson and J. M. Olefsky, "The origins and drivers of insulin resistance," Cell, vol. 152, no. 4, pp. 673-684, 2013.

[8] G. M. Reaven, "Insulin resistance: the link between obesity and cardiovascular disease," Medical Clinics of North America, vol. 95, no. 5, pp. 875-892, 2011.

[9] L. Cacciagiú, A. I. González, A. Elbert et al., "Do insulin resistance conditions further impair the lipid and inflammatory profile in end-stage renal disease patients on hemodialysis?" Metabolic Syndrome and Related Disorders, vol. 12, no. 4, pp. 220-226, 2014.

[10] E. Siew and T. Ikizler, "Determinants of insulin resistance and its effects on protein metabolism in patients with advanced chronic kidney disease," Contributions to Nephrology, vol. 161, pp. 138144, 2008.

[11] A. Borazan and D. N. Binici, "Relationship between insulin resistance and inflamation markers in hemodialysis patients," Renal Failure, vol. 32, no. 2, pp. 198-202, 2010.

[12] S. Kurat, H. B. Colak, A. Toraman, H. Tekçe, C. Ulman, and O. Bayturan, "Relationship of insulin resistance in chronic haemodialysis patients with inflammatory indicators, malnutrition, echocardiographic parameters and 24 hour ambulatory blood pressure monitoring," Scandinavian Journal of Urology and Nephrology, vol. 44, no. 4, pp. 257-264, 2010.
[13] A. Fragoso, F. Mendes, A. P. Silva, and P. L. Neves, "Insulin resistance as a predictor of cardiovascular morbidity and endstage renal disease," Journal of Diabetes and its Complications, vol. 29, no. 8, pp. 1098-1104, 2015.

[14] K. Shinohara, T. Shoji, M. Emoto et al., "Insulin resistance as an independent predictor of cardiovascular mortality in patients with end-stage renal disease," Journal of the American Society of Nephrology, vol. 13, no. 7, pp. 1894-1900, 2002.

[15] Y. Li, L. Zhang, Y. Gu, C. Hao, and T. Zhu, "Insulin resistance as a predictor of cardiovascular disease in patients on peritoneal dialysis," Peritoneal Dialysis International, vol. 33, no. 4, pp. 411418, 2013.

[16] Y. Zhou, Z. Yu, H. Jia et al., "Association between insulin resistance and carotid arterial stiffness in nondiabetic hemodialysis patients," Blood Purification, vol. 28, no. 3, pp. 193-199, 2009.

[17] Y.-C. Lin, C.-Y. Hsu, C.-C. Kao et al., "Incidence and prevalence of ESRD in Taiwan renal registry data system (TWRDS): 20052012," Acta Nephrologica, vol. 28, no. 2, pp. 65-68, 2014.

[18] A. M. Hung and T. A. Ikizler, "Factors determining insulin resistance in chronic hemodialysis patients," Contributions to Nephrology, vol. 171, pp. 127-134, 2011.

[19] D. Teta, "Insulin resistance as a therapeutic target for chronic kidney disease," Journal of Renal Nutrition, vol. 25, no. 2, pp. 226-229, 2015.

[20] T. J. Arneson, J. Liu, Y. Qiu, D. T. Gilbertson, R. N. Foley, and A. J. Collins, "Hospital treatment for fluid overload in the medicare hemodialysis population," Clinical Journal of the American Society of Nephrology, vol. 5, no. 6, pp. 1054-1063, 2010.

[21] J. L. Merino, P. Domínguez, B. Bueno, Y. Amézquita, B. Espejo, and V. Paraíso, "Application of model of incremental haemodialysis, based on residual renal function, at the initiation of renal replacement therapy," Nefrología, vol. 37, no. 1, pp. 3946, 2017.

[22] T. V. Duong, T. Wong, C. Su et al., "Associations of dietary macronutrients and micronutrients with the traditional and nontraditional risk factors for cardiovascular disease among hemodialysis patients," Medicine, vol. 97, no. 26, p. el1306, 2018.

[23] T. V. Duong, T. Wong, H. Chen et al., "The cut-off values of dietary energy intake for determining metabolic syndrome in hemodialysis patients: A clinical cross-sectional study," PLoS ONE, vol. 13, no. 3, p. e0193742, 2018.

[24] T.-C. Wong, H.-Y. Su, Y.-T. Chen et al., "Ratio of C-reactive protein to albumin predicts muscle mass in adult patients undergoing hemodialysis," PLoS ONE, vol. 11, no. 10, Article ID e0165403, 2016.

[25] Y.-F. Chiu, Y.-C. Chen, P.-Y. Wu et al., "Association between the hemodialysis eating index and risk factors of cardiovascular disease in hemodialysis patients," Journal of Renal Nutrition, vol. 24, no. 3, pp. 163-171, 2014.

[26] D. R. Matthews, J. P. Hosker, A. S. Rudenski, B. A. Naylor, D. F. Treacher, and R. C. Turner, "Homeostasis model assessment: insulin resistance and $\beta$-cell function from fasting plasma glucose and insulin concentrations in man," Diabetologia, vol. 28, no. 7, pp. 412-419, 1985.

[27] S. J. Moon, J. E. Lee, J.-K. Kim et al., "The relationship between hemodialysis modality and insulin resistance in non-diabetic hemodialysis patients," Blood Purification, vol. 39, no. 1-3, pp. 224-229, 2015.

[28] M. J. Sarnak and A. S. Levey, "Cardiovascular disease and chronic renal disease: a new paradigm," American Journal of Kidney Diseases, vol. 35, no. 4, pp. S117-S131, 2000. 
[29] P. Yerram, P. R. Karuparthi, L. Hesemann, J. Horst, and A. Whaley-Connell, "Chronic kidney disease and cardiovascular risk," Journal of the American Society of Hypertension, vol. 1, no. 3, pp. 178-184, 2007.

[30] The National Kidney Foundation Kidney Disease Outcomes Quality Initiative (K/DOQI) Workgroup, "K/DOQI clinical practice guidelines for cardiovascular disease in dialysis patients," American Journal of Kidney Diseases, vol. 45, supplement 3, no. 4, pp. 16-153, 2005.

[31] Expert Panel on Detection Evaluation and Treatment of High Blood Cholesterol in Adults, "Executive summary of the third report of the national cholesterol education program (NCEP) expert panel on detection, evaluation, and treatment of high blood cholesterol in adults (adult treatment panel III)," Journal of the American Medical Association, vol. 285, no. 19, pp. 24862497, 2001.

[32] KDOQI National Kidney Foundation, "Clinical practice guidelines and clinical practice recommendations for anemia in chronic kidney disease in adults," American Journal of Kidney Diseases, vol. 47, Supplement 3, pp. S16-S85, 2006.

[33] A. A. Portale, "Blood calcium, phosphorus, and magnesium," in Primer on the Metabolic Bone Diseases and Disorders of Mineral Metabolism. 4, M. J. Favus, Ed., pp. 115-118, Williams \& Wilkins, Philadelphia, Pennsylvania, 1999.

[34] Kidney Disease: Improving Global Outcomes (KDIGO) CKD Work Group, "KDIGO clinical practice guideline for the diagnosis, evaluation, prevention, and treatment of chronic kidney disease-mineral and bone disorder (CKD-MBD)," Kidney International, vol. 76, Supplement 113, pp. S1-S130, 2009.

[35] S. D. de Ferranti and N. Rifai, "C-reactive protein: a nontraditional serum marker of cardiovascular risk," Cardiovascular Pathology, vol. 16, no. 1, pp. 14-21, 2007.

[36] A. A. Lopes, J. L. Bragg-Gresham, S. J. Elder et al., "Independent and joint associations of nutritional status indicators with mortality risk among chronic hemodialysis patients in the dialysis outcomes and practice patterns study (DOPPS)," Journal of Renal Nutrition, vol. 20, no. 4, pp. 224-234, 2010.

[37] C. P. Kovesdy, D. L. Regidor, R. Mehrotra et al., "Serum and dialysate potassium concentrations and survival in hemodialysis patients," Clinical Journal of the American Society of Nephrology, vol. 2, no. 5, pp. 999-1007, 2007.

[38] A. Sicras-Mainar, E. Ruíz-Beato, R. Navarro-Artieda, and J. Maurino, "Comorbidity and metabolic syndrome in patients with multiple sclerosis from Asturias and Catalonia, Spain," BMC Neurology, vol. 17, no. 1, p. 134, 2017.

[39] B. Hildrum, A. Mykletun, T. Hole, K. Midthjell, and A. A. Dahl, "Age-specific prevalence of the metabolic syndrome defined by the international diabetes federation and the national cholesterol education program: The Norwegian HUNT 2 study," BMC Public Health, vol. 7, pp. 220-229, 2007.

[40] L. Razzouk and P. Muntner, "Ethnic, gender, and age-related differences in patients with the metabolic syndrome," Current Hypertension Reports, vol. 11, no. 2, pp. 127-132, 2009.

[41] S. Shahrokh, P. Heydarian, F. Ahmadi, F. Saddadi, and E. Razeghi, "Association of inflammatory biomarkers with metabolic syndrome in hemodialysis patients," Renal Failure, vol. 34, no. 9, pp. 1109-1113, 2012.

[42] T. A. Lakka and D. E. Laaksonen, "Physical activity in prevention and treatment of the metabolic syndrome," Applied Physiology, Nutrition, and Metabolism, vol. 32, no. 1, pp. 76-88, 2007.
[43] H. D. Do, V. Lohsoonthorn, W. Jiamjarasrangsi, S. Lertmaharit, and M. A. Williams, "Prevalence of insulin resistance and its relationship with cardiovascular disease risk factors among Thai adults over 35 years old," Diabetes Research and Clinical Practice, vol. 89, no. 3, pp. 303-308, 2010.

[44] A. S. Soto González, D. B. Guerrero, M. B. Soto, S. P. Díaz, M. Martinez-Olmos, and O. Vidal, "Metabolic syndrome, insulin resistance and the inflammation markers $\mathrm{C}$-reactive protein and ferritin," European Journal of Clinical Nutrition, vol. 60, no. 6, pp. 802-809, 2006.

[45] Y. Arslan and G. Kiziltan, "Nutrition-related cardiovascular risk factors in hemodialysis patients," Journal of Renal Nutrition, vol. 20, no. 3, pp. 185-192, 2010.

[46] Y. Liu, W.-C. Lee, B.-C. Cheng, Lung-Chih Li et al., "Association between the achievement of target range CKD-MBD markers and mortality in prevalent hemodialysis patients in Taiwan by using the kidney disease: improving global outcomes clinical guidelines," BioMed Research International, vol. 2016, Article ID 1523124, 7 pages, 2016.

[47] S. Cheng, "Factors impacting quality of life in dialysis patients," Cheng Ching Mededical Journal, vol. 5, no. 2, pp. 31-40, 2009.

[48] S. E. Liebman, S. P. Lamontagne, L.-S. Huang, S. Messing, and D. A. Bushinsky, "Smoking in dialysis patients: a systematic review and meta-analysis of mortality and cardiovascular morbidity," American Journal of Kidney Diseases, vol. 58, no. 2, pp. 257-265, 2011. 


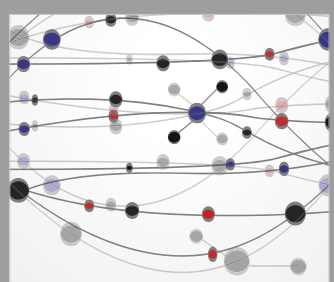

The Scientific World Journal
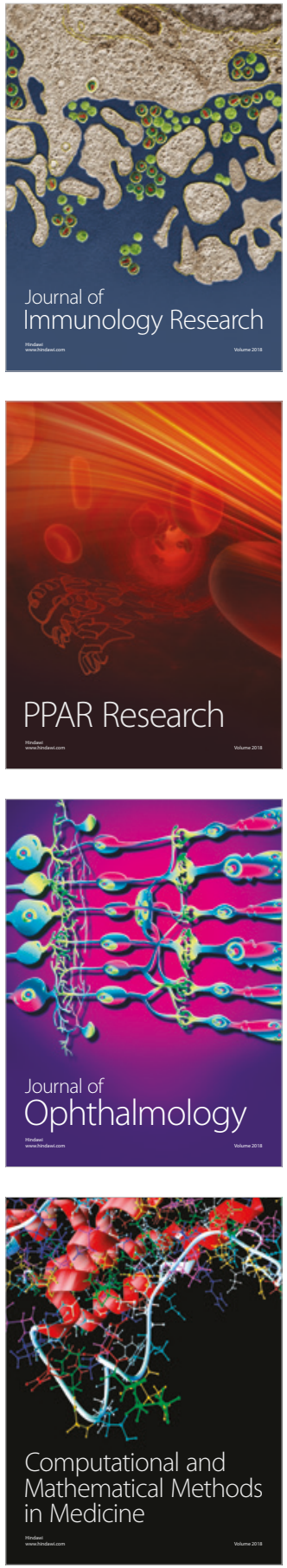

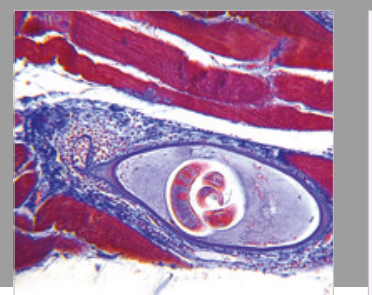

Gastroenterology Research and Practice

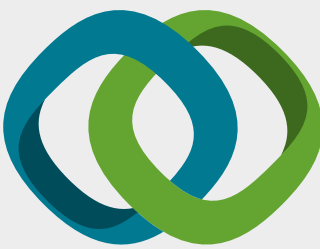

\section{Hindawi}

Submit your manuscripts at

www.hindawi.com
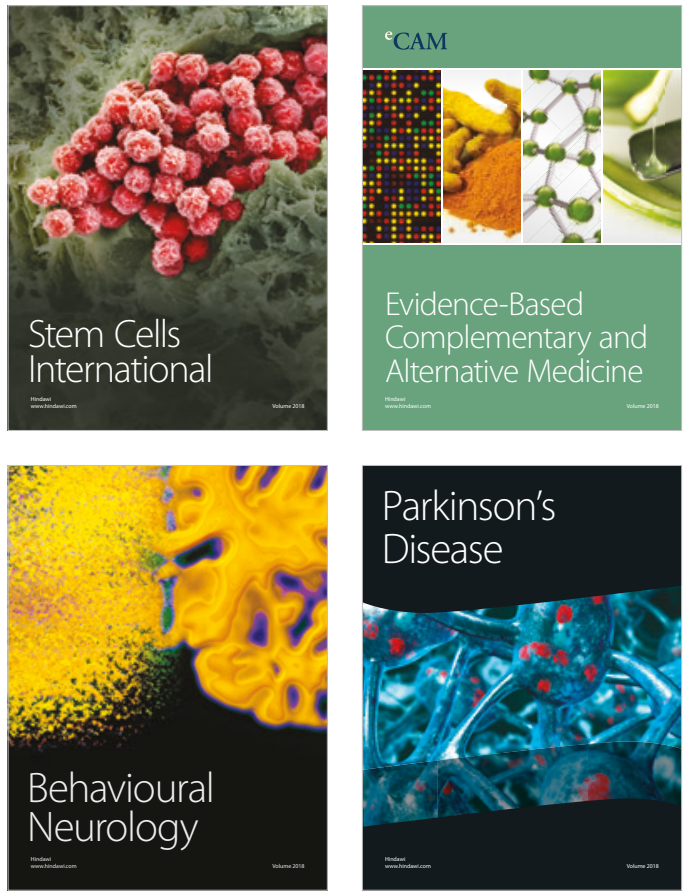

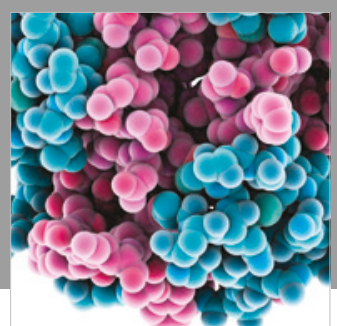

ournal of

Diabetes Research

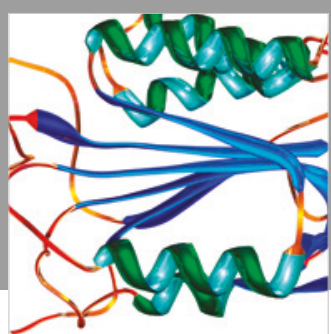

Disease Markers
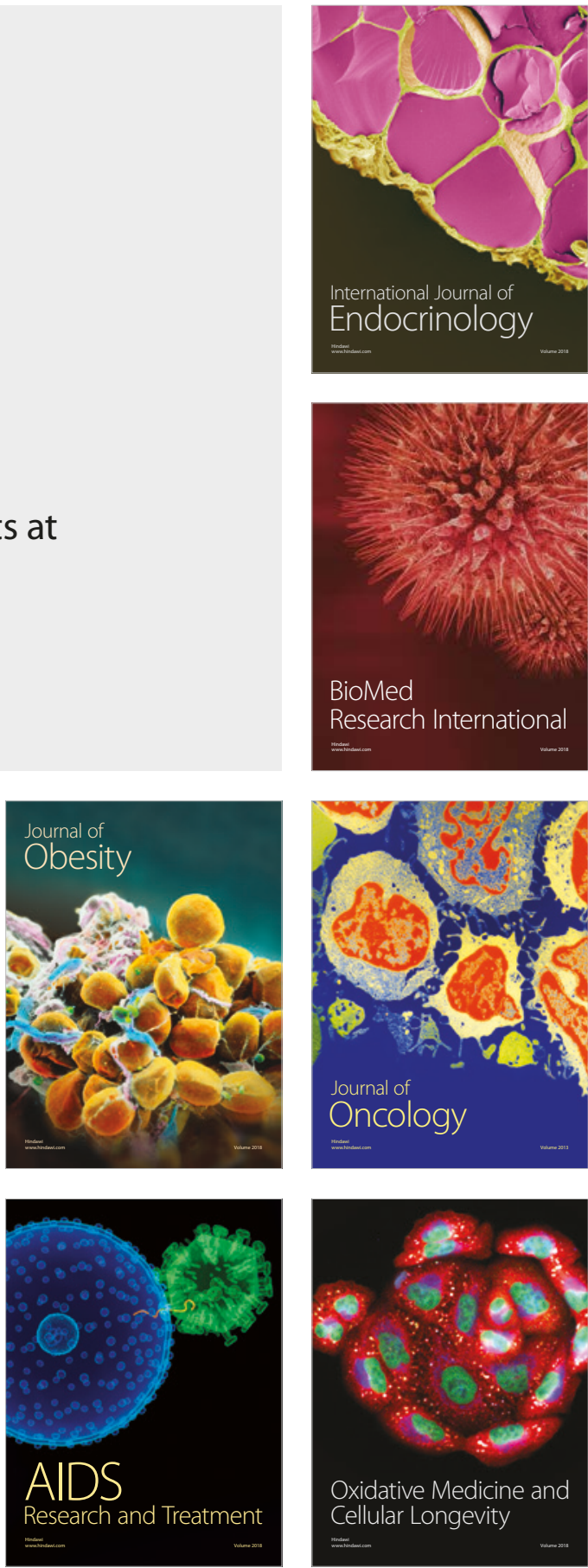